\title{
Balint-Holmes syndrome due to stroke following SARS-CoV-2 infection: a single-case report
}

\author{
Francesco Panico ${ }^{1}$ (D) $\cdot$ Angela Arini ${ }^{2} \cdot$ Pierluigi Cantone $^{2} \cdot$ Claudio Crisci $^{2} \cdot$ Luigi Trojano $^{1}$
}

Received: 17 September 2020 / Accepted: 24 October 2020 / Published online: 27 October 2020

(C) Fondazione Società Italiana di Neurologia 2020

\section{Dear Editor-in-Chief,}

Balint-Holmes' syndrome (BHS) is a neuropsychological condition related to bilateral lesions in the parieto-occipital cortex, characterized by simultanagnosia, ocular apraxia, and optic ataxia [1,2]. Simultanagnosia is the inability to detect multiple visually presented objects (dorsal type) and to recognize the individual parts of a multipart object (ventral type). Ocular apraxia is an impairment in saccade initiation and visual pursuit, despite unrestricted ocular movements. Optic ataxia is the difficulty in performing movements directed to visual objects, in the absence of primary sensory and motor disorders.

BHS has been described mainly after cerebrovascular events, but also in relation to HIV encephalopathy, carbon monoxide poisoning, or posterior cortical atrophy $[1,2]$.

Here we report a patient showing BHS following bilateral parieto-occipital damage due to a stroke after SARS-CoV-2 infection.

A 55-year-old male teacher, with systemic arterial hypertension under pharmacological treatment (ramipril and amlodipine) and mild hypercholesterolemia, suddenly developed fever, cough, dyspnea, ageusia, and anosmia in March 2020. After detection of SARS-CoV-2 viral nucleic acid by a nasopharyngeal swab, the diagnosis of coronavirus disease 2019 (COVID-19) was made. In a few days, the patient was admitted to intensive care unit for worsening of respiratory distress.

First authorship is shared by Francesco Panico and Angela Arini

Francesco Panico

francesco.panico@unicampania.it

1 Department of Psychology, University of Campania "Luigi Vanvitelli”, Viale Ellittico 31, 81100 Caserta, Italy

2 Clinic Center Rehabilitation Institute, Viale Maria Bakunin 171, 80126 Naples, Italy
At discharge from intensive care unit, one month later (April 16, 2020), DG presented with tetraplegia and vision loss. A magnetic resonance scan showed temporo-parietooccipital cortico-subcortical lesions in the right hemisphere and parieto-occipital cortico-subcortical lesions in the left hemisphere (Fig. 1) which were compatible with a bilateral stroke in the territory of the inferior division of the middle cerebral artery.

When the patient was transferred to a rehabilitation unit (May 2020), he was alert and cooperative, and showed mild bilateral hyposthenia with hyporeflexia, mild tactile sensory loss, and left homonymous hemianopia. The neuropsychological examination at bedside revealed left spatial neglect (Fig. 2a) associated with word finding difficulties and mild executive dysfunction.

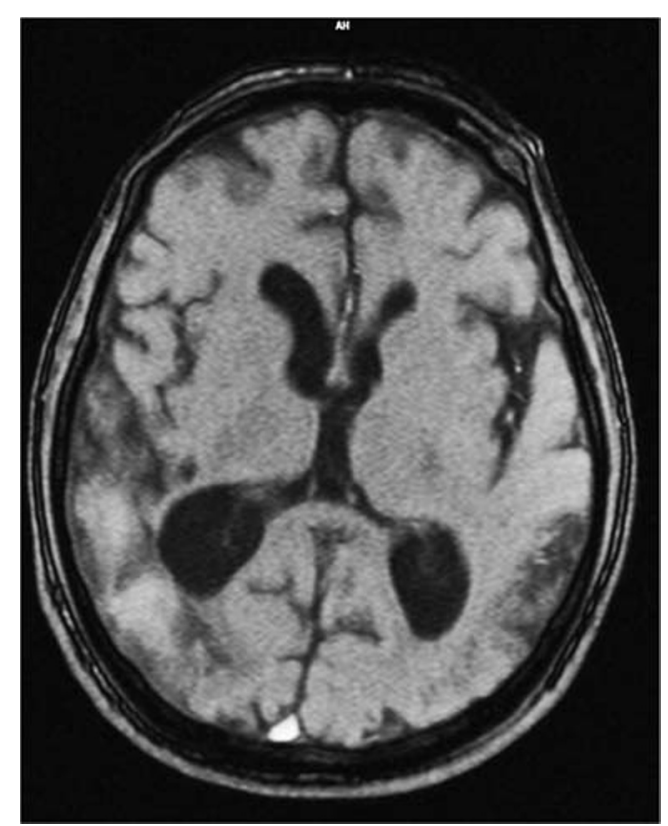

Fig. 1 Axial brain T1-MR scan showing the wide right temporo-parietal and the left parietal lesions 


\section{a}

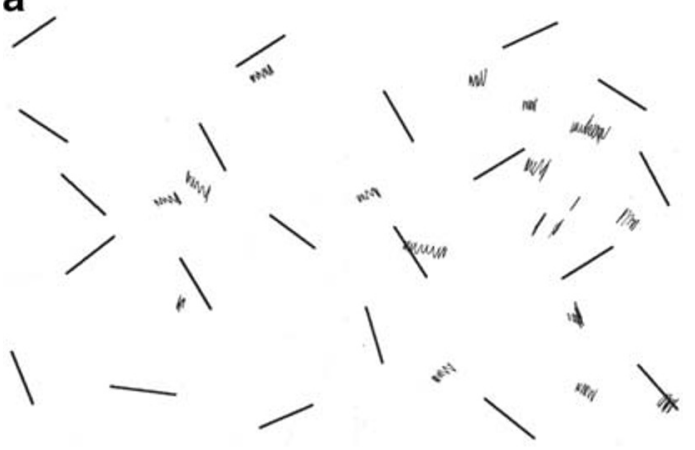

C

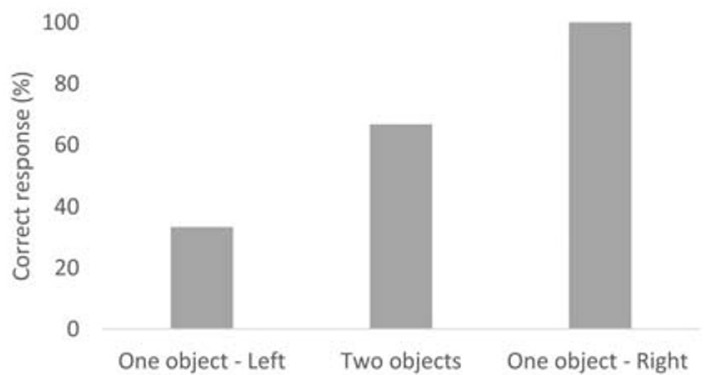

d

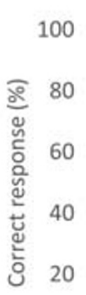

0
Gaze Apraxia - Static test

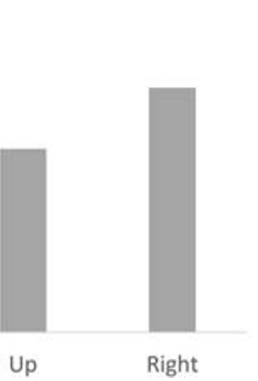

Fig. 2 a Patient's performance in a line cancellation test. b Percentage of correct responses in a task to assess optic ataxia: the patient was asked to reach for a visually displayed object in the left (LF), right (RF), or central (C) visual fields by using his left (LH) or right (RH) hand. c Left, percentage of correct response in a task to assess dorsal simultanagnosia: the patient had to look for a visually presented object in the left or right visual fields, or two objects displayed simultaneously at the center. Right, ventral simultanagnosia: images containing both local features (parts) and global features (the whole) were shown to the patient (e.g., a large letter "G" formed by small letters "E," or a big square whose sides were made

When required to reach for visually displayed objects, the patient revealed marked impairments, mainly in reaching for objects located in his left visual field with his left and right hands, and in his right field with his left hand (Fig. 2b). This behavior was consistent with the classical descriptions of optic ataxia [2].

The patients also showed obvious impairments in the visual perception domain, as he failed to recognize large silhouettes made up of small parts; moreover, on some occasions, the patient could not identify two objects

b

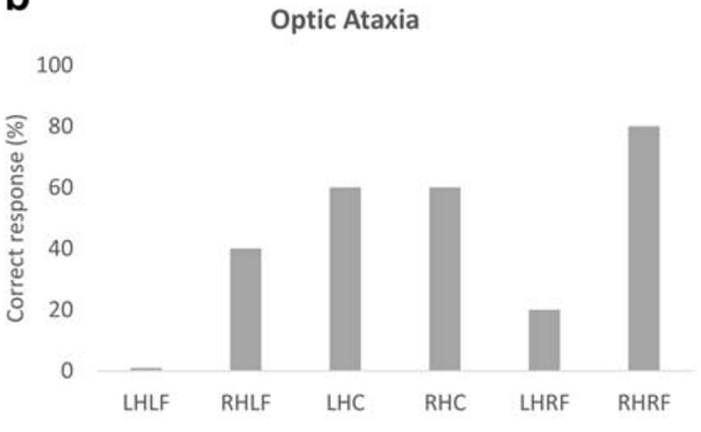

Simultanagnosia - Ventral form

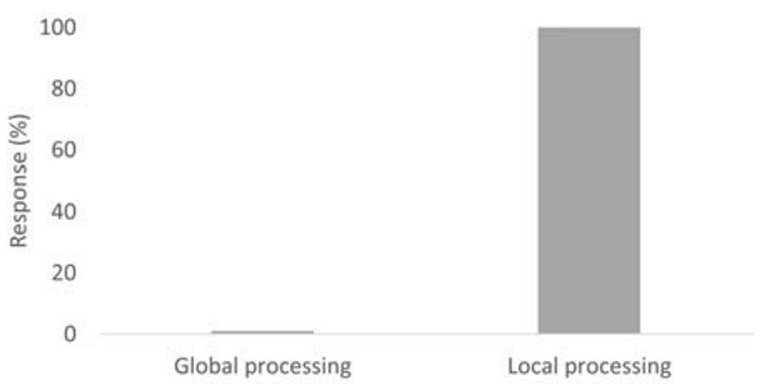

Gaze Apraxia - Dynamic test

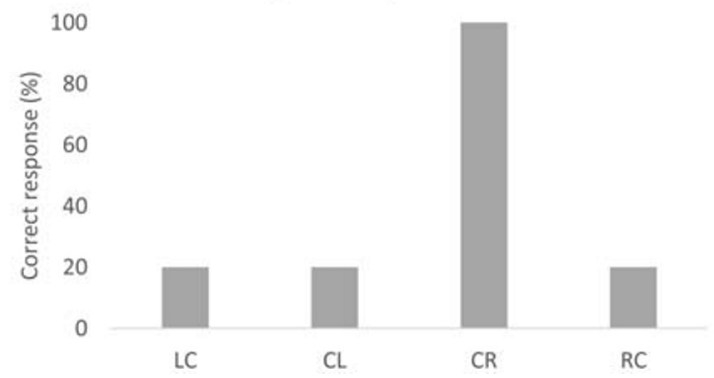

by small circles); the response corresponds to the local or global features reported by the patient. d Percentage of correct responses in two tasks assessing gaze apraxia: left, static test - an object was presented in four possible locations (left, right, up, down); the patient had to move the eyes toward the object with no head movement. Right, dynamic test - an object was slowly moved by the experimenter from left to center (LC), center to left (CL), center to right (CR), or right to center (RC) on the horizontal axis; the patient had to follow the object with his eyes with no head movements

simultaneously presented in his central visual field (Fig. 2c). These findings demonstrated the presence of both ventral and (to a small degree) dorsal simultanagnosia, combined with left visual hemineglect.

The clinical picture was also characterized by impairments in static and dynamic tests assessing the ability to direct gaze toward specific targets. The patient proved to be unable to move his eyes toward statically presented objects, particularly left-located objects, without making concurrent head movements; moreover, he could not gaze at an object slowly 
moving along the horizontal axis (Fig. 2d). These tasks thus revealed severely impaired saccade initiation and visual pursuit (ocular apraxia).

All these clinical characteristics constitute the full-blown picture and are pathognomonic of BHS, closely resembling the original descriptions by Balint, with a main involvement of peripheral visual fields and of the left-hand movements in peripheral vision [2].

To our knowledge, this is the first case report of BHS following bilateral parieto-temporo-occipital stroke correlated with SARS-CoV-2 cerebral vasculopathy.

As several studies demonstrated that SARS-CoV-2 infection can affect the central nervous system [3-5], we could consider it as a likely causal factor in DG. Presence of vascular risk factors in our patient is consistent with recent evidence suggesting that acute cerebrovascular events related to COVID-19 are more frequent in patients with severe respiratory distress and common cardiovascular risk factors [3]. Ageusia and anosmia are further relevant signs of nervous system involvement in SARS-CoV-2 infection [4].

The exact pathophysiological mechanisms of the association between SARS-CoV-2 and stroke have to be fully comprehended [3-5]. Literature suggests that such an association could be mediated by misdirected immune response inducing coagulopathy and vasoconstriction. Systemic inflammation and the potential direct action of the virus may cause endothelial dysfunction, resulting in a hypercoagulable state and consequent ischemic strokes [3].

Our observations are in line with recent studies on the impact of SARS-CoV-2 infection on the central nervous system. As distribution of strokes related to COVID-19 has not been analyzed yet, our report underlines that they can determine even quite rare neurological syndromes, such as BHS, for which SARS-CoV-2 infection might represent a possible risk factor.
Acknowledgments We would like to express our gratitude to Dr. Francesca Russo for providing us the patients' clinical data and for support during the assessment.

Author contributions FP and AA collected the data and wrote the first draft. LT, CC, and PC revised the original manuscript. All authors read and approved the final manuscript.

Funding FP received funding by the program V:ALERE2019 Università della Campania “Luigi Vanvitelli,” D.R. 907/2019, prot. n. 148008, 04/ $10 / 2019$

\section{Compliance with ethical standards}

Conflict of interest The authors have no relevant financial or nonfinancial relationships to disclose.

Ethical approval Written informed consent was obtained from the patient; the experiment was performed in accordance with the ethical standards laid down in the 1964 Declaration of Helsinki and its later amendments.

\section{References}

1. Hecaen H, de Ajuriaguerra J (1954) Balint's syndrome (psychic paralysis of visual fixation) and its minor forms. Brain 77:373-400

2. Pisella L, Rossetti Y, Rode G (2017) Optic ataxia in Bálint-Holmes syndrome. Ann Phys Rehabil Med 60(3):148-154. https://doi.org/ 10.1016/j.rehab.2016.01.003

3. Matos AR, Quintas-Neves M, Oliveira AI, Dias L, Marques S, Carvalho R, Alves JN (2020) COVID-19 associated central nervous system vasculopathy [published online ahead of print, 2020 Jun 2]. Can J Neurol Sci:1-6. https://doi.org/10.1017/cjn.2020.109

4. Sultana S, Ananthapur V (2020) COVID-19 and its impact on neurological manifestations and mental health: the present scenario. Neurol Sci 41:3015-3020. https://doi.org/10.1007/s10072-02004695-w

5. Niazkar HR, Zibaee B, Nasimi A, Bahri N (2020) The neurological manifestations of COVID-19: a review article. Neurol Sci 41:16671671. https://doi.org/10.1007/s10072-020-04486-3

Publisher's note Springer Nature remains neutral with regard to jurisdictional claims in published maps and institutional affiliations. 